\title{
24 Months follow-up of diastemas closure using ceramic veneers: a case report
}

\begin{abstract}
As patients' aesthetic expectations are increasing, diastema closure are frequently requested. Advances in ceramic material and bonding techniques helps the dentists to restore function and aesthetic with conservative and biologically sound methods as well as promoting longterm oral health. Critical planning and clinical care should be carried in accordance with interrelationship between the teeth, soft tissues, lip and face. This case report focuses on the effectiveness of using wax-up as a diagnostic aid to fabricate mock-up in aesthetic dental treatment cases.
\end{abstract}

Volume 8 Issue 6 - 2017

\author{
Mayyadah Almozainy \\ Department of Restorative Dentistry, King Saud University, \\ Saudi Arabia
}

\begin{abstract}
Correspondence: Mayyadah Almozainy, Department of Restorative Dentistry, King Saud University, P.O.Box 2456, Riyadh I I45I, Saudi Arabia, Email mmozainy@yahoo.com
\end{abstract}

Received: October 30, 2017| Published: November 15, 2017

\section{Introduction}

As patients' aesthetic expectations are increasing, diastema closure are frequently requested. Many treatment modalities can be used to close diastemas, depending on the etiology and the patient preference. Direct composite restorations have their limitations aesthetically in cases with multiple large spaces between the teeth, as well as short survival rate in comparison to porcelain laminate veneers (PLVs), where the failure rate of (PLVs) have been reported as only $0 \%$ to $5 \%$ over one to five years. ${ }^{1}$ Porcelain laminate veneers offers many advantages over direct composite restorations, such as color and shape stability, abrasion resistance, ability to match the shade and glossiness of natural teeth and low plaque accumulation, they offer high physical properties such as compressive strength. ${ }^{2-4}$ Since the introduction of (PLVs) in the early 1980's, they are considered as the primary mode of restorations in aesthetic dentistry. ${ }^{1}$ Advances in ceramic material and bonding techniques help the dentists to restore function and aesthetic with conservative and biologically sound methods as well as promoting long-term oral health. Critical planning and clinical care should be carried in accordance with interrelationship between the teeth, soft tissues, lip and face. Harmony and symmetry with dentofacial elements should be also respected since the primary indication of (PLVs) is to improve aesthetics. ${ }^{5,6}$ This case report focuses on the effectiveness of using wax-up as a diagnostic aid to fabricate mockup in aesthetic dental treatment cases. Since this technique plays an important role in treatment planning, in controlling the function and aesthetic of the end result before teeth preparation, it will also ease the communication between dentist-patient and dental technician. Also it will act as guidance for conservative minimally invasive teeth preparation and in fabrication of temporary restoration. It is very important that through this way, we gain the trust of the patient. As from the first step of work, the patient feels involved in the decisionmaking and will have a realistic expectation of the final treatment outcome.

\section{Case reports}

A 35 years old female patient presented to restorative clinic at the dental college, King Saud University, her chef complaint was the multiple spaces between her upper front teeth and she expressed her desire to receive (PLVs) in specific to adjust the shape and color of her teeth. Detailed clinical examination was performed; digital photographs and X-rays were taken. After discussing the treatment options with the patient. Porcelain laminate veneers were the treatment of choice for the maxillary anterior teeth in this case because there are large and multiple spaces between teeth. For the mandibular teeth in office bleaching was considered since they are in good shape and alignment using 35\% hydrogen peroxide (Zoom! whitespeed, PhilipsUSA) only for the mandibular teeth since they are in a good shape and alignment (Figure1), subsequently alginate impressions (United Alginate, Major Prodotti Dentari SpA, Moncalieri, Italy) were taken for diagnostic models. The models were studied to decide the shape and size of the restorations with help of a diagnostic wax up. On the following appointment, the wax up model was presented to the patient (Figure 2). With the use of Silicon putty mock-ups that were fabricated directly over the patient teeth based on the new smile design.

The patient was given the chance to see her new smile design over her teeth, where she liked the new shape and size of her teeth. Then, the teeth were prepared over the mock-ups to ensure minimal invasion to the natural teeth structure. Before proceeding for teeth preparation, shade was selected using Vitapan Classical shade guide (Vita Zahnfabrik, Germany) and it was confirmed with the patient as BL3. The maxillary teeth preparation was done after two weeks of bleaching of the lower arch. Preparation started from the right first premolar to the left first premolar to receive porcelain laminate veneers. However, the right second premolar was prepared to receive full ceramic crown due to the fact that it is endodontically treated with weak remaining tooth structure. The teeth preparation for veneers were kept in enamel at a depth of $0.5 \mathrm{~mm}$ using a depth cutting diamond and a tapered diamond $1 \mathrm{~mm}$ in diameter (DIATECH, Dübendorf, Switzerland). $0.25 \mathrm{~mm}$ chamfer was maintained in the cervical region (Figure 3). The chamfer finish lines were kept at the level of gingival margin. The preparation was finalized using fine diamond finishing bur (DIATECH, Dübendorf, Switzerland) and sof-lex discs (3M, USA). The centric stops were carefully avoided during preparing the palatal finish line. The proximal preparation was extended beyond the contact area to avoid visibility of the tooth restoration junction and to give more freedom to the lab technician to divide the present spaces as needed between the (PLVs) during fabrication. Gingival retraction 
cords were placed (\#00, Ultradent, South Jordan UT, USA) to exposed the gingival margins and to ensure final impression accuracy which was taken as a single step with prefabricated plastic tray using silicon impression material (Aquasil impression material, Dentsply, International Inc. Canada). Provisional veneers were fabricated directly in the clinic with the silicon putty mold that was taken over the wax-up using Protemp 4 provisional material (3M ESPE St. Paul, MN, and USA), excess material was removed using surgical blade No.12, finishing and polishing was performed to the temporary veneers and care was taken to open the gingival embrasures to ensure good gingival health during the cementation visit (Figure 4). The lab order was filled with all the details needed plus the selected shade, and the (PLVs) were fabricated using (IPS e.max Press, Ivoclar Vivadent Schaan, Liechtenstein, Zurich) (Figure 5).

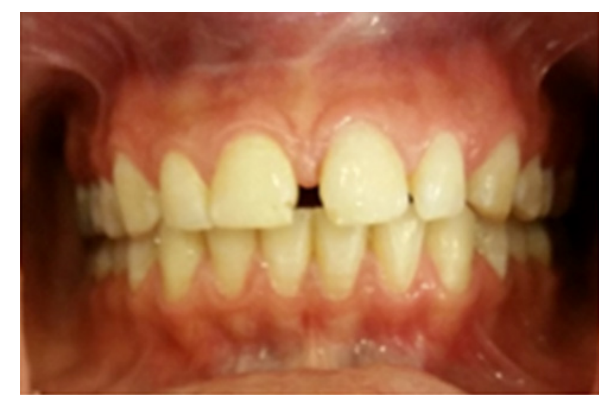

Figure I Preoperative intraoral frontal view.

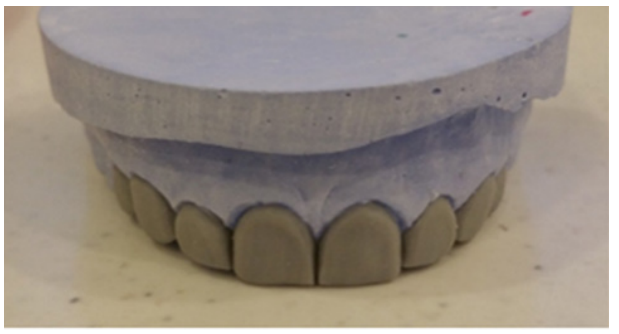

Figure 2 Wax up model.

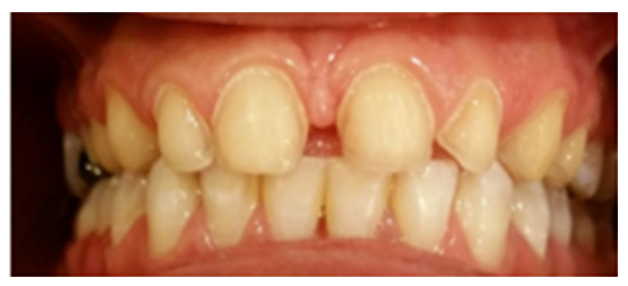

Figure 3 Frontal view of teeth preparation.

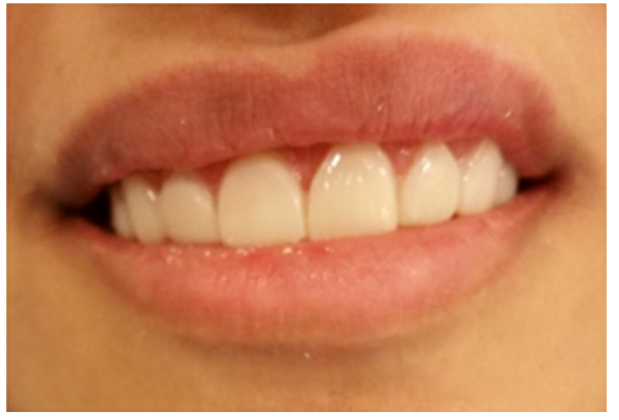

Figure 4 Temporary veneers.

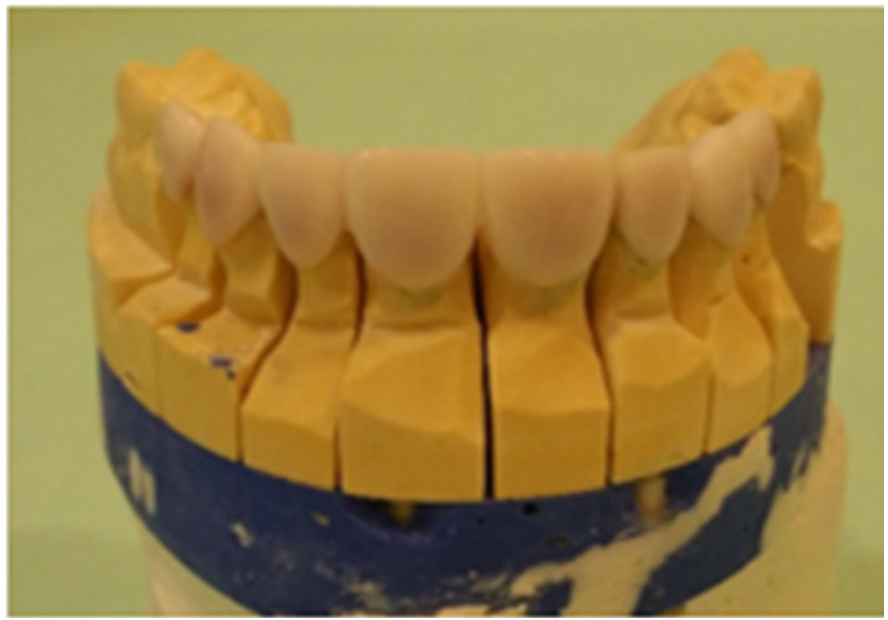

Figure $\mathbf{5}$ Ceramic veneers on the cast.

\section{Veneers try in and cementation}

On the next visit, the temporary veneers and crown were removed and the teeth were cleaned. Each veneers was tried individually to check marginal adaptation and fit, then all the veneers as well as the crown were placed together to check the proximal contact and harmony between them in relation to each other and the gingival margins. The patient was given the chance to see them and she expressed her satisfaction. The cementation was done with translucent shade dual cure resin cement Veriolink N (Ivoclar Vivadent, Schaan, Lichtenstein) following the manufacturer recommendations, the eternal surface of the ceramic restorations were etched using $9.5 \%$ hydrofluoric acid for 30 seconds (Condicionador de Porcelanas, Dentsply Brasil). After thorough washing with water and drying, silane coupling agent (Monobond Plus, Ivoclar Vivadent) was applied on the internal surface aswell and left to react for 60 seconds and then air dried. The teeth structure was etched with $37 \%$ phosphoric acid for 30 seconds (Total Etch, Ivoclar Vivadent, Schaan, and Lichtenstein) then washed for another 30s and gently dried. Adhesive agent (ExciTE F DSC, Ivoclar Vivadent, Schaan, and Lichtenstein) was placed over the prepared teeth and the inner surfaces of the ceramics and the applied layer was thinned using air spray to remove excess. The catalyst and base of the scent was mixed and applied on the fitting surface of the ceramics, the veneers were seated simultaneously starting from the centrals followed by laterals, canines, first premolars on each side and lastly the crown on the right second premolar. Tuck curing for one second using LED-curing unit (Blue phase LED-curing light, Ivoclar Vivadent, Schaan, Lichtenstein) was done and the excess cement on the margins was removed using microbrush and explorer and the excess cement interproximally was removed using thin dental floss, after that the curing process was completed for 20 seconds for each tooth. Occlusal contacts were checked in centric, protrusive and lateral movements and high contact points were removed using fine diamond finishing bur. The final result is shown on Figures 6-10. At 24 months recall visit, the overall restored teeth were in good condition with good gingival heath, the patient was satisfied with the treatment provided and she was advised to schedule a bleaching visit for the lower arch to maintain the shade matching with the upper arch veneers (Figures 11,12). 


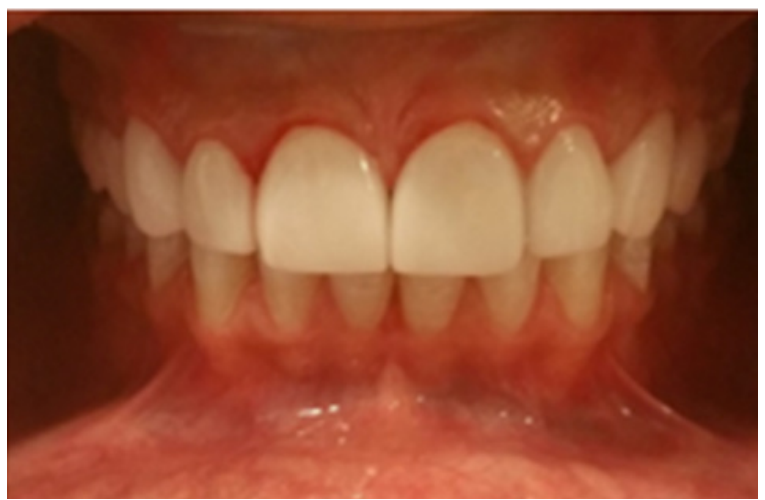

Figure 6 Frontal view after cementation.

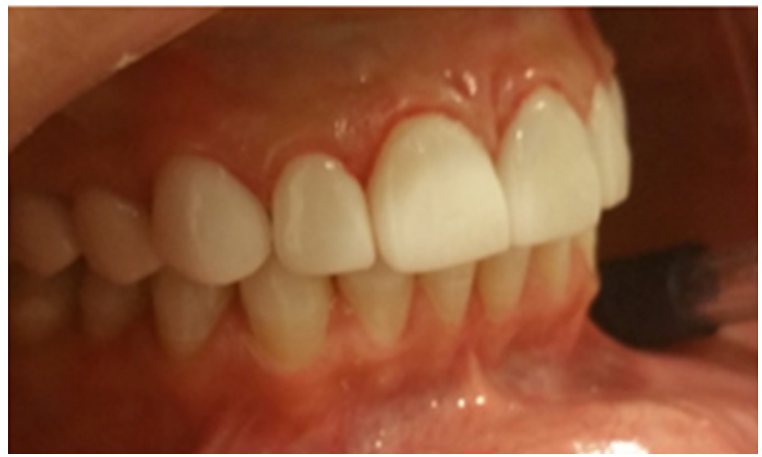

Figure 7 Lateral view after cementation.

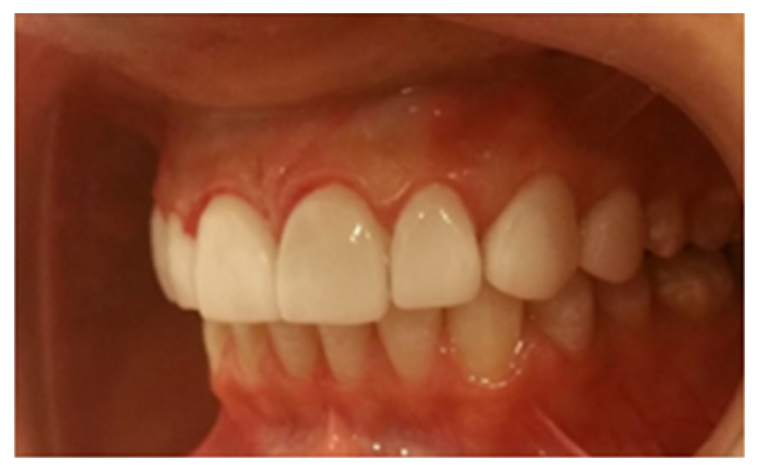

Figure 8 Lateral view after cementation.

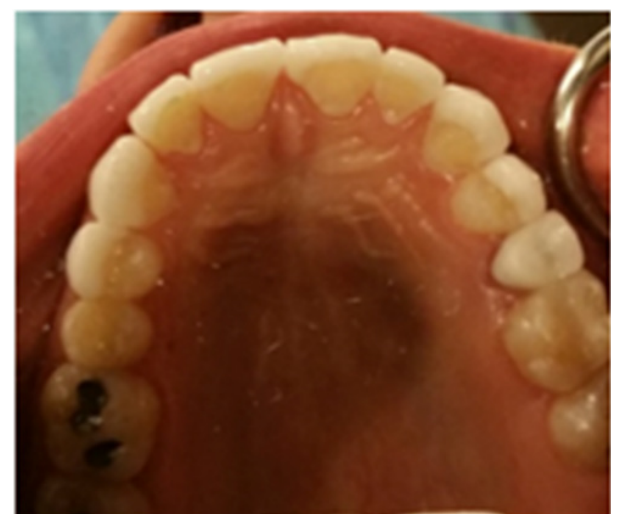

Figure 9 fitting surface after cementation.

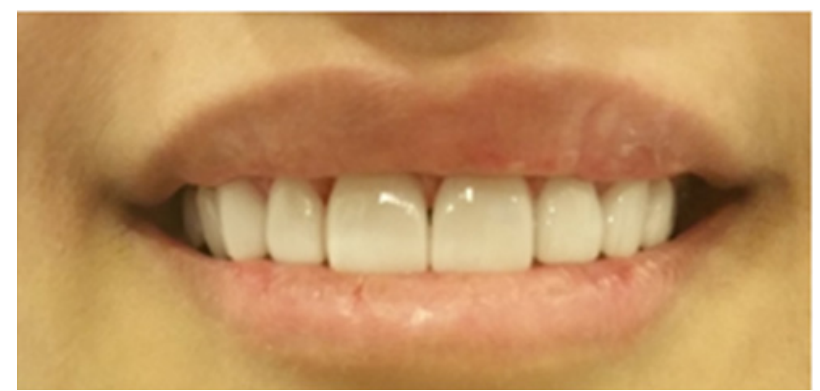

Figure 10 Patient's smile after cementation.

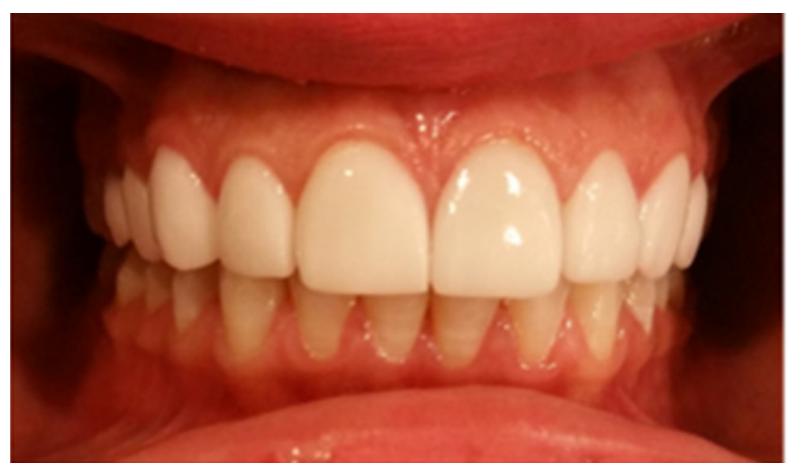

Figure II Recall visit after 24 months.

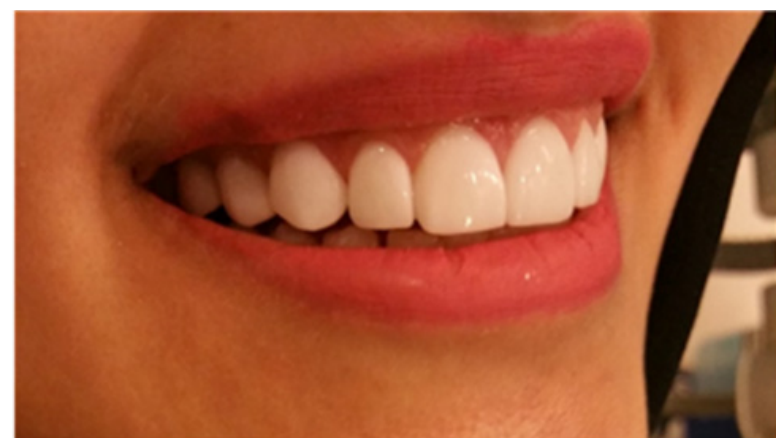

Figure 12 Patient's smile (24 months recall visit).

\section{Discussion}

Many patients are seeking aesthetic treatment with a desire to improve their smile, one of the common issues that they are concerned about diastemas between the teeth. In order to achieve a satisfying long term functional treatment result rather than just filling the spaces, precise diagnosis of the causative factor of diastema should be explored. Since there are many factors that can be attributed as an etiology of diastema, such as: microdontia, discrepancy between teeth and jaw size, high frenum attachment, periodontal disease, teeth loss, developmental problems or habits. ${ }^{7}$ Treatment options for diastema closure can vary between orthodontic treatment, restorative treatment, surgical periodontal treatment or combination of them depending on the etiology of the diastema. ${ }^{8}$ If restorative treatment is the treatment of choice, it can be achieved by using many options such as: direct or indirect composite veneers, porcelain laminate veneers or full crowns. ${ }^{8,9}$

Precise planning and smile design are the key factors to successful aesthetic outcome in (PLVs) cases, diagnostic wax-up used to 
fabricate mock-ups which are used after as an accurate preparation guide to prevent over or under teeth reduction. ${ }^{10}$ In the presented case, teeth were prepared through the resin mock-up that represent the definitive teeth contours and allows a minimally invasive uniform reduction. Recall visits are very important after completion of treatment to evaluate the status of the case. In 24 months recall visit of the presented case, the periodontal response was outstanding due to fact that cervical margins were kept supragingival as recommended. ${ }^{11}$

\section{Conclusion}

This article describes the treatment of maxillary anterior teeth with porcelain veneers, using a systemic practical conservative approach that gave the chance to the dentist and the patient to visualize the predictable final outcome and agree on it prior to actual teeth preparation. Excellent aesthetic results can be achieved with minimal reduction due to high physical properties of (PLVs) in addition to the bonding ability of luting resins used for cementation.

\section{Funding}

None.

\section{Acknowledgments}

None.

\section{Conflicts of interest}

None.

\section{References}

1. Peumans M1, Van Meerbeek B, Lambrechts P. Porcelain veneers: a review of the literature. J Dent. 2000;28(3):163-177.
2. Seghi RR, Sorensen JA. Relative flexural strength of six new ceramic materials. Int J Prosthodont. 1995;8(3):239-246.

3. McLaren EA. All-ceramic alternatives to conventional metal-ceramic restorations. Compend Contin Educ Dent. 1998;19(3):307-326.

4. Rotoli BT, Lima DA, Pini NP, et al. Porcelain veneers as an alternative for esthetic treatment: clinical report. Oper Dent.2013; 38(5):459-466.

5. Bichacho N. Porcelain laminates: integrated concepts in treating diverse aesthetic defects. Pract Periodontics Aesthet Dent. 1995;7(3): 13-23.

6. Magne P, Belser Urs. Bonded porcelain restorations in the anterior dentition: a biomimetic approach. Quintessence Publishing Co, USA, 2002; p. 1-406.

7. Oesterle LJ, Shellhart WC. Maxillary midline diastemas: A look at the causes. J Am Dent Assoc. 1999;130(1):85-94.

8. Goldstein RE. Restorative treatment of Diastema. In: Decker B, Hoywood VB, editors. Esthetic in Dentistry. Volume 2: Individual Teeth, Missing Teeth, Malocclusion, Facial Appearance, 2nd ed. B.C. Decker Inc, Canada. 2002; p. 1-884.

9. Rammelsberg P. Clinical performance of metal-free polymer crowns after 3 years in service. $J$ Dent. 2005;517-523.

10. Simon H, Magne P. Clinically based diagnostic wax-up for optimal esthetics: the diagnostic mock-up. J Calif Dent Assoc. 2008;36(5):355362.

11. Magne P, Belser UC. Novel porcelain laminate preparation approach driven by a diagnostic mock-up. J Esthet Restor Dent. 2004;16(1):7-18. 